\title{
Development of a Distributed Representative Human Model Generation and Analysis System for Multiple-Size Product Design
}

\author{
Baekhee Lee ${ }^{1}$, Kihyo Jung ${ }^{2}$, Heecheon You ${ }^{1}$ \\ ${ }^{1}$ Department of Industrial and Management Engineering, POSTECH, Pohang, 790-784 \\ ${ }^{2}$ School of Industrial Engineering, University of Ulsan, Ulsan, 680-749
}

\begin{abstract}
Objective: The aim of this study is to develop a distributed representative human model(DRHM) generation and analysis system. Background: DRHMs are used for a product with multiple-size categories such as clothing and shoes. It is not easy for a product designer to explore an optimal sizing system by applying various distributed methods because of their complexity and time demand. Method: Studies related to DRHM generation were reviewed and the RHM generation interfaces of three digital human model simulation systems $\left(\mathrm{Jack}^{\circledR}\right.$, RAMSIS $^{\circledR}$, and CATIA Human $\left.{ }^{\circledR}\right)$ were reviewed. Results: DRHM generation steps are implemented by providing sophisticated interfaces which offer various statistical techniques and visualization methods with ease. Conclusion: The DRHM system can analyze the multivariate accommodation percentage of a sizing system, provide body sizes of generated DRHMs, and visualize generated grids and DRHMs. Application: The DRHM generation and analysis system can be of great use to determine an optimal sizing system for a multiple-size product by comparing various sizing system candidates.
\end{abstract}

Keywords: Multiple-size product design, Sizing system, Distributed representative human model

\section{Introduction}

Digital human model(DHM) simulation system(예: $\mathrm{Jack}^{\circledR}, \mathrm{RAMSIS}^{\circledR}$ ) 은 가상환경상에서 대표인체모델(representative human model, RHM)을 생성하여 인간공학적인 제품과 작업공간의 설계 및 평가를 위한 효율적인 도구로 사용되고 있다. RHM은 제품 설계대상인구의 인체크기를 통계적으로 적합하게 대표하는 소수의 인체모델로서, 효율 적인 제품 설계 및 개발을 위해 필수적이다(Jung and You, 2007). 예를 들면, Park et al.(2008) 과 Lee et al.(2010) 은 소수의 RHM을 기반으로 한국형 헬리콥터 조종실과 방사성폐기물처리장 주제어실을 인간공학적으로 설계하고
평가하기 위하여 DHM simulation system을 활용하였다 (Figure 1).

DHM simulation system의 RHM 생성은 percentile 방 법과 custom-built 방법 등에 의해 수행될 수 있는데 각각 설계대상인구에 대한 RHM의 생성 효율성 측면의 한계점을 지니고 있다. Percentile 방법은 대부분 대표적으로 사용되 는 3 가지 $\operatorname{RHM}\left(5^{\text {th }}, 50^{\text {th }}\right.$, 그리고 $95^{\text {th }}$ percentiles $)$ 을 제공 하며, 두 개 이상의 인체변수에 대한 인구수용비율은 목표수 용비율보다 저하된다(HFES 300, 2004). 또한, custombuilt 방법은 인체변수(예: 26 개, $\mathrm{Jack}^{\circledR}$ )별로 RHM의 인체 크기를 사용자가 직접 입력하는 인터페이스를 제공하고 있 는데, 생성 목적에 맞는 인체변수들의 크기를 결정하고 사용 자가 직접 입력하는데 많은 시간이 소요된다.

Corresponding Author: Heecheon You. Department of Industrial and Management Engineering, POSTECH, Pohang, 790-784.

Phone: 054-279-2210, E-mail: hcyou@postech.ac.kr

Copyright@2011 by Ergonomics Society of Korea(pISSN:1229-1684 eISSN:2093-8462). All right reserved. 


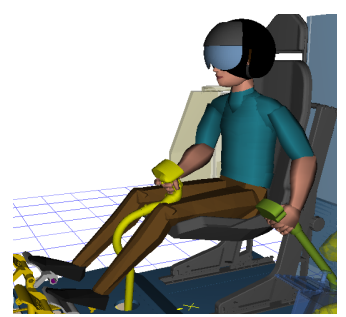

(a) Helicopter cockpit (Park et al., 2008)

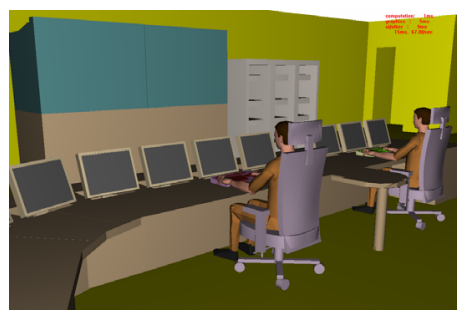

(b) Radioactive waste facility control room(Lee et al., 2010)
Figure 1. Ergonomic product design using a digital human model simulation system

RHM 인체크기 결정을 위한 RHM 생성 방법들 중 산포 대표인체모델(distributed representative human model, DRHM) 의 생성은 의복과 같은 다중치수제품 (multiplesize product)의 대량맞춤생산을 위한 치수체계 개발에 활 용되고 있다. 그러나, DRHM을 생성하고 대표성 (representativeness) 분석에 특화된 시스템의 개발은 미흡한 실 정이다. 따라서, DRHM을 생성하고 분석하는 시스템의 개발 은 인간공학적인 다중치수제품 설계 시 비용 절감, 시간 단 축, 그리고 최적 치수체계 선정에 기여할 수 있다.

본 연구는 다중치수제품 설계를 위한 DRHM 생성 및 분석 시스템을 개발하였다. 본 연구에서는 문헌조사를 통 해 DRHM 생성 절차 및 기법을 조사하고, 기존 DHM simulation system의 DRHM 생성 인터페이스의 특성 및 한계점을 파악하였다. 그리고, DRHM 생성 절차 및 방법을 적용하여 DRHM 생성 및 분석에 특화된 시스템을 개발하 였다.

\section{Literature Review}

DRHM은 치수체계가 있는 제품(예: 장갑)의 설계 및 평 가 시에 생성된다. DRHM은 제품 설계대상인구 전반에 지 정된 비율(예: 95\%)을 수용하도록 격자들을 배치한 후, 각 격자에서 한 개씩 DRHM을 선정하여 생성된다(Jung et al., 2010). DRHM 생성 절차는 3 단계(중요변수 선정, distributed method 결정, 그리고 DRHM 인체크기 결정)로 구분되는데, Figure 2 와 같이 각 단계에서는 다양한 통계적 기법(예: regression analysis)이 사용된다(Jung, 2009).

본 연구는 세 가지 대표적인 DHM simulation system들 (Jack, RAMSIS, 그리고 CATIA Human)에 대하여 특성 및 한계점을 파악하였다(Table 1). 예를 들어, Jack은 US Army 인체측정 data (Gordon et al., 1988)를 기반으로 성 별(female, male) 및 percentile $\left(1^{\text {st }}, 5^{\text {th }}, 50^{\text {th }}, 95^{\text {th }}, 99^{\text {th }}\right)$ 을 선택하여 RHM을 생성하는 인터페이스를 제공하고 있으 나, 성별 및 다양한 연령대를 특정 비율로 혼합하여 RHM을 생성하는 기능을 제공하지 않는 것으로 파악되었다.

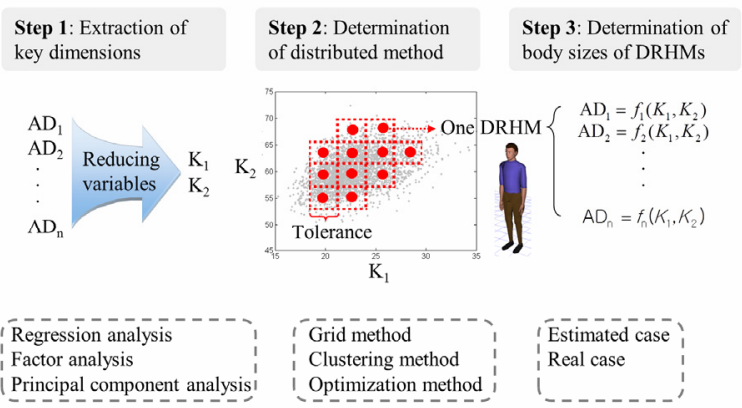

Note: $\mathrm{AD}=$ anthropometric dimension, $\mathrm{K}=$ key dimension

Figure 2. Distributed representative human model(DRHM) generation process \& used statistical techniques (AD: anthropometric dimension, $\mathrm{K}$ : key dimension)

Table 1. Characteristics of distributed representative human model generation interfaces of digital human model simulation systems

\begin{tabular}{c|c|c|c}
\hline Factors & $\begin{array}{c}\text { Jack } \\
\text { (SIMENS) }\end{array}$ & $\begin{array}{c}\text { RAMSIS } \\
\text { (Human Solutions) }\end{array}$ & $\begin{array}{c}\text { CATIA Human } \\
\text { (Dassault Systemes) }\end{array}$ \\
\hline $\begin{array}{c}\text { Database } \\
\text { Nation }\end{array}$ & $\begin{array}{c}\text { US Army } \\
(1988)\end{array}$ & $\begin{array}{c}\text { Germany etc., } \\
17 \text { nations } \\
(1984 \sim 2020)\end{array}$ & $\begin{array}{c}\text { American etc., } \\
5 \text { nations } \\
(* \text { N.S. })\end{array}$ \\
\hline Gender & Female, Male & Female, Male & Female, Male \\
\hline Age groups & $* *$ N/A & $\begin{array}{c}\text { Fixed 4 groups } \\
(\text { e.g., 30 49) }\end{array}$ & N/A \\
\hline Number of AD & 26 & 24 & N/A \\
\hline $\begin{array}{c}\text { RHM } \\
\text { Generation } \\
\text { method }\end{array}$ & $\begin{array}{c}\text { Percentile } \\
\text { Custom-built }\end{array}$ & $\begin{array}{c}\text { Percentile } \\
\text { Custom-built }\end{array}$ & Percentile \\
\hline * N.S.: not specified &
\end{tabular}

\section{System Development}

본 연구는 Microsoft Visual Studio C\# 2008과 MATLAB 2008a(ver. 7.6)을 활용하여 DRHM을 생성하 고 분석하는 시스템을 개발하였다. 본 시스템은 Figure 3 과 같이 DRHM 생성 절차 5 단계가 구현된 입력 인터페이스와 생성된 DRHM에 대한 분석 결과 및 시각화된 결과를 제공 한다. 


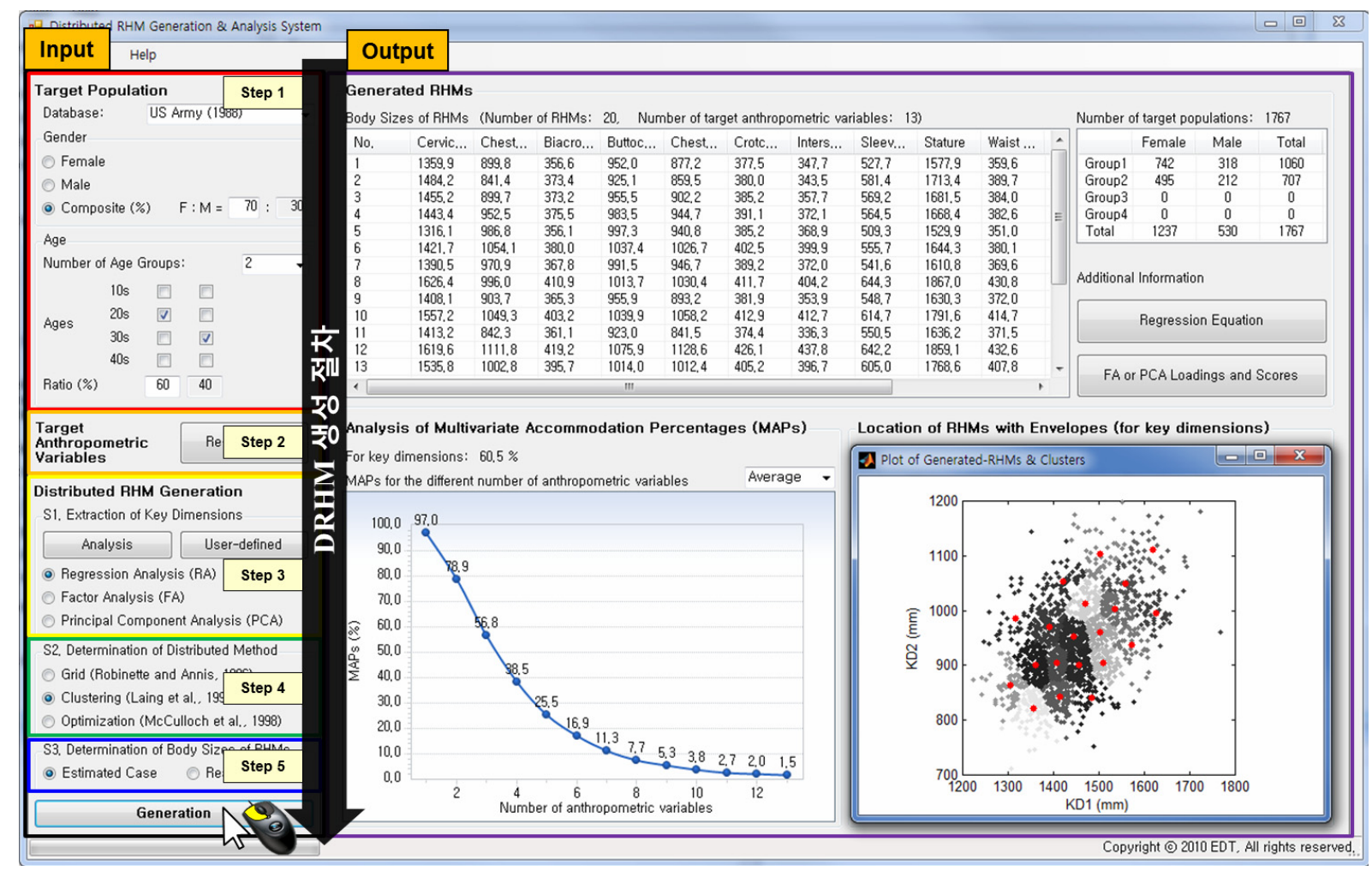

Figure 3. System overview

\subsection{DRHM generation}

\subsubsection{Step 1: Target population selection}

설계대상인구는 인체측정 data로부터 성별과 연령대를 선 택하여 선정된다. 본 시스템은 여러 인체측정 database(US Army, 1998; US Army Pilot, 1998; Korean Pilot, 2007) 와 연동되도록 구축되어 있으며, 남녀 비율과 10 대부터 40 대까지 각 연령대 비율을 지정하여 설계대상인구를 형성할 수 있도록 하였다.

\subsubsection{Step 2: Target anthropometric variable selection}

본 시스템은 설계대상 인체변수들을 대분류, 소분류, 그리 고 치수유형으로 분류하여 사용자가 용이하게 선택할 수 있 도록 하였다. 예를 들어, Figure 4와 같이 가슴둘레 (chest circumference) 는 trunk (대분류), chest(소분류), circumference(치수유형) 순으로 탐색하여 선정된다.

\subsubsection{Step 3: Extraction of key dimensions}

중요변수 추출단계는 대표적으로 사용되는 3 가지 통계적 분석 방법(regression analysis, factor analysis, principal component analysis)을 적용할 수 있는 각각의 인터페이스 를 제공한다.

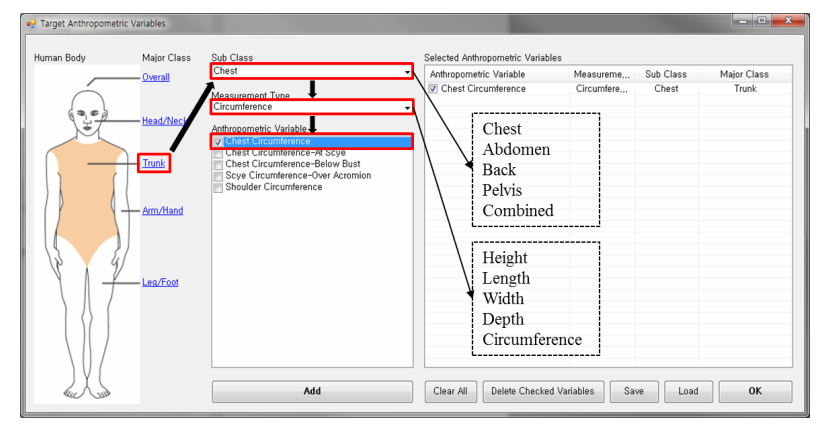

Figure 4. Interface of target anthropometric variable selection

예를 들어, regression analysis 인터페이스는 Figure 5 와 같이 선택된 설계대상 인체변수들로부터 중요변수 개수 (1 5개)에 따른 중요변수 후보와 나머지 변수간의 평균수 정회귀계수의 경향을 graph 및 table로 제공한다. 또한, 사 용자가 중요변수를 사전에 알고 있을 경우 'User-defined' 버튼을 사용하여 직접 선정할 수 있다.

\subsubsection{Step 4: Determination of distributed method}

DRHM 생성 방법 선정단계에서는 3가지 DRHM 생성 방 법(grid, cluster, optimization method)을 적용할 수 있는 
각각의 인터페이스를 제공한다. 예를 들어, grid method 인 터페이스는 Figure 6 과 같이 선정된 중요변수들의 다양한 기술적 통계치(예: mean, percentile)를 제공하고(Kwon et al., 2009), 생성될 grid의 설계허용공차를 인체변수마다 동 일한 혹은 다른 값을 설정할 수 있으며, 목표수용비율이나 각 격자의 최소인구수용비율을 선택할 수 있도록 구현되었다.

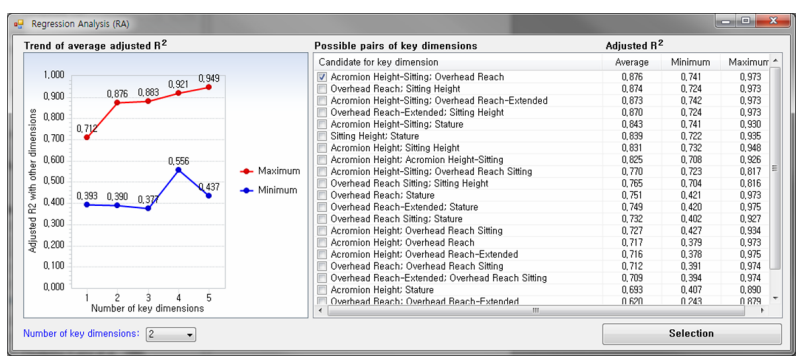

Figure 5. Interface of regression analysis

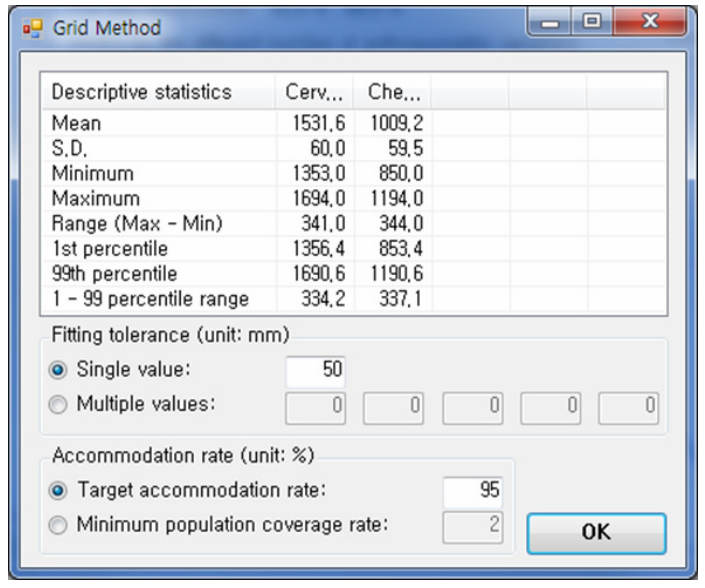

Figure 6. Interface of grid method

\subsubsection{Step 5: Determination of DRHM's body sizes}

DRHM 인체크기 결정단계에서는 중요변수 측면에서 DRHM 생성 방법에 의해 생성된 격자들을 대표할 수 있는 두 가지 인체크기 선정 방법(estimated case 및 real case) 이 제공된다(Figure 7). Estimated case 사용 방법은 생성 된 격자의 형상학적 중심(centroid)에서 DRHM을 결정 하는 반면, real case 사용 방법은 centroid에서 Euclidian distance가 가장 최소인 실제 case를 DRHM으로 선정한다.

\subsection{DRHM analysis}

본 시스템은 생성된 DRHM의 인체크기 정보, 수용비율
분석 결과, 그리고 관련 정보의 시각화된 기능을 제공한다 (Figure 3). 본 시스템은 생성된 DRHM의 설계대상 인체 변수별 인체크기를 table로 제공한다. 본 시스템은 생성된 $\mathrm{DRHM}$ 에 대하여 중요변수 측면의 인구수용비율과 선정된 인 체변수 개수에 따른 5 가지 (mean, $\mathrm{SD}$, minimum, maximum, median) 단일수용비율 및 다변량수용비율 분석 기능을 제 공한다. 본 시스템은 생성된 DRHM에 대하여 중요변수 측 면에서 생성된 격자와 DRHM의 시각화 정보를 제공한다.

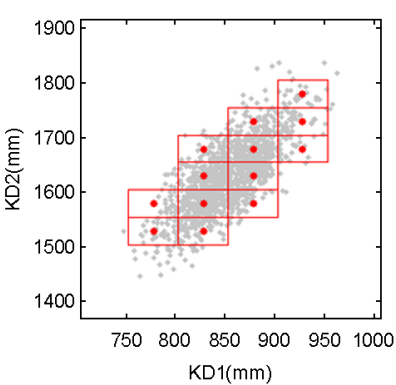

(a) Estimated case

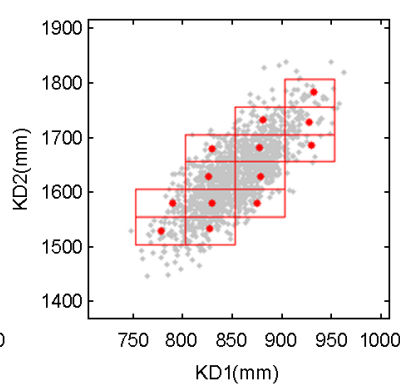

(b) Real case
Figure 7. Determination of the body sizes of distributed representative human models(illustrated)

\section{Discussion}

본 연구에서는 다중치수제품 설계를 위한 DRHM 생성 및 분석에 특화된 시스템이 개발되었다. 본 시스템은 5 단계 DRHM 생성 절차(설계대상인구 선정, 설계대상 인체변수 선정, 중요변수 추출, distributed method 선정, 그리고 $\mathrm{DRHM}$ 인체크기 결정)를 적용하여 사용자가 DRHM 생성 을 용이하고 신속하게 할 수 있도록 제공하였다. 본 시스템 은 DRHM 생성에 사용되는 통계적 기법을 총체적으로 제공 하여 사용자가 원하는 맞춤형 치수체계 설계가 가능하도록 인터페이스를 제공하였다. 본 시스템은 생성된 DRHM 분석 에 특화된 시스템으로서 DRHM의 인체크기, 인구수용비율 분석, 그리고 시각화된 정보를 제공하여, 인간공학적 제품의 치수체계 개발 시 유용하게 활용될 수 있다.

본 시스템은 설계 가능한 치수체계를 용이하고 신속하게 분석함으로써 체계적인 비교 분석을 통한 최적 치수체계 설계 시 활용될 수 있다. 기존 DRHM 생성관련 연구들은 $\mathrm{DRHM}$ 생성 시 체계적인 방법간의 비교 없이 연구자가 임 의로 선정한 방법을 적용하여 제품의 치수체계를 설계한 것 으로 파악되었다. 반면, 본 연구에서 개발된 시스템을 활용 
하면 치수체계 설계자는 DRHM 생성 절차 별로 적용되는 다양한 기법들을 적용하여 치수체계 대안들을 파악하고 이 들을 비교 분석함으로써 최적 치수체계를 선정할 수 있다.

본 연구에서 개발된 시스템은 주변부 대표인체모델 (boundary representative human model, BRHM) 생성 및 분석 시스템의 개발과 함께 DHM simulation system의 custom-built RHM 생성 인터페이스와의 연동이 필요하다. $\mathrm{BRHM}$ 은 자동차와 같은 단일치수제품 (one-size product) 의 인간공학적 설계 시 적용되는데 본 연구의 DRHM 생성 및 분석 시스템과 같은 특화된 시스템으로 개발되어 생성 될 수 있다. 또한, 개발된 시스템을 통해 생성된 RHM들이 DHM simulation system의 custom-built RHM 생성 기능 과 연동되어 인간공학적 평가(예: 도달성, 여유공간 등)가 수 행될 수 있도록 하는 시스템간 상호 운용(interoperability) 에 대한 연구가 필요하다.

\section{Acknowledgements}

This research was supported by the Basic Science Research Program through the National Research Foundation of Korea (NRF) funded by the Ministry of Education, Science, and Technology(2010-0028229).

\section{References}

Chaffin, D. B., Improving digital human modeling for proactive ergonomics in design. Ergonomics, 48(5), 478-491, 2005.

Gordon, C., et al. 1988 Anthropometric Survey of US Army Personnel: Methods and Summary Statistics, Technical Report NATICK/TR-89/ 044, 1988.

HFES 300, Guidelines for Using Anthropometric Data in Product Design, Santa Monica, California: Human Factors and Ergonomics Society, 2004.

Jung, K., Kwon, O. and You, H., Development of a digital human model generation method for ergonomic design in virtual environment, International Journal of Industrial Ergonomics, 39(5), 744-748, 2009.

Jung, K., Development of a Multivariate Representative Human Model generation Method for Anthropometric Design, Unpublished Ph.D. dissertation, Pohang University of Science and Technology, Pohang, Korea, 2009.

Jung, K., You, H. and Kwon, O., Evaluation of the multivariate accommodation performance of the grid method, Applied Ergonomics, 42,
$156-161,2010$.

Kwon, O., Jung, K., You, H. and Kim H., Determination of key dimensions for a glove sizing system by analyzing the relationship between hand dimensions. Applied Ergonomics, 40, 762-766, 2009.

Laing, R. M., Holland, E. J. and Niven, B. E., Development of sizing systems for protective clothing for the adult male, Ergonomics, 42(10), 1249-1257, 1999.

Lee, B., Chang, Y., Jung, K., Jung, I. and You, H, Ergonomic evaluation of a control room design of radioactive waste facility using digital human simulation, Journal of the Ergonomics Society of Korea, 29(3), 383-391, 2010.

McCulloch, C. E., Paal, B. and Ashdown, S. P., An optimization approach to apparel sizing, Journal of the Operational Research Society, 49, 492-499, 1998.

Park, J., Jung, K., Lee, W., Kang, B., Lee, J., Eom, J., Park, S. and You, H., Development of an ergonomic assessment method of helicopter cockpit using digital human simulation, In Proceedings of the 2008 Spring Conference of the Ergonomics Society of Korea, 2008.

Robinette, K. M. and Annis, J. F., A Nine-Size System for Chemical Defense Gloves (Technical Report AAMRL-TR-86-029), Ohio: Wright-Patterson Air Force Base, 1986.

Rosenblad-Wallin, E., An anthropometric study as the basis for sizing anatomically designed mittens, Applied Ergonomics, 18(4), 329-333, 1987.

Zheng, R., Yu, W. and Fan, J., Development of a new Chinese bra sizing system based on breast anthropometric measurements, International Journal of Industrial Ergonomics, 37, 697-705, 2007.

\section{Author listings}

Baekhee Lee: x200won@postech.ac.kr

Highest degree: M.S., Department of Industrial Engineering, POSTECH Position title: Ph.D., Department of Industrial Engineering, POSTECH Areas of interest: Ergonomic Product Design \& Development, Digital Human Modeling \& Simulation, Vehicle Ergonomic

Kihyo Jung: kjung@ulsan.ac.kr

Highest degree: Ph.D., Department of Industrial and Management Engineering, POSTECH

Position title: Assistant Professor, University of Ulsan

Areas of interest: Ergonomic Product Design, Digital Human Modeling and Simulation, Usability Testing, Musculoskeletal Disorders Prevention 
Heecheon You: hcyou@postech.ac.kr

Highest degree: Ph.D., Department of Industrial Engineering, The Pennsylvania State University

Position title: Professor, Department of Industrial Engineering, POSTECH

Areas of interest: Ergonomic Product Design \& Development, User

Interface Design \& Evaluation, Digital Human Modeling \& Simulation,

Human Performance \& Workload Assessment, Usability Testing
Date Received : 2011-01-05

Date Revised : 2011-07-29

Date Accepted : 2011-07-30 